Parent participation in the nursing process significantly contributes to the overall wellbeing of a newborn and the whole family. It is important to ensure that nurses, who take care of newborns and collaborate with their family members, are well-acquainted with the influence that an infant's illness and critical condition as well as the stress have on the functioning of a family unit

The Aim To compare parents' and nurses' perceptions of parents needs in Neonatal Intensive Care Unit.

The study was conducted in Kaunas University Hospital NICU. The sample consisted of 181 parents and 37 nurses. The NICU Family Needs Inventory was used for data collection. The instrument included five following subscales of needs: proximity, information, assurance, support and comfort. Reliability of the scale was identified as 0.94 using Cronbach's $\alpha$.

The parents' needs perceived by both groups were compared within the subscales. Using Mann'o-Whitney rank test the significant differences between the parents' and nurses' assessments of individual statements were identified. The majority of statistically significant differences between parents and nurses have been found in case of assessment of proximity (75.0\%) and informational $(72.7 \%)$ needs related to the possibility for parents to be close to their newborn and to receive timely and adequate information about their newborn's health condition. Parents, if compared to nurses, assessed these needs as more important. The assessment of support needs showed the lowest number of differences $(38.9 \%)$. There was a statistically significant difference in case of the decision making needs related to the newborn care that parents identified as being more important. Nurses perceived as being more important the needs related to parent-toparent support and possibility to share feelings.

The comparison of parents and nurses perceptions of parents needs in NICU showed that there are statistically significant differences of individual statements assessment within the all five subscales.

\section{PS-262 RISK PROFILE TO PREVENT RE-ADMITTANCE TO THE NEONATAL INTENSIVE CARE UNIT}

LE Smit, MJ Hemmink, JM Wielenga. IC Neonatology, Emma Children's Hospital/Academic Medical Center, Amsterdam, Netherlands

\subsection{6/archdischild-2014-307384.562}

Background and aims Infants at the Neonatology Intensive Care Unit (NICU) of the Emma Children's Hospital/Academic Medical Centre Amsterdam are transferred to regional hospitals once they no longer need intensive care. Unfortunately, a number of these infants are re-admitted to the NICU. This study investigates the similarities between these hospitalised infants, in order to develop a way to limit the number of infants re-admitted to the NICU.

Methods The first step in the process is to classify different diagnoses for re-admittance of infants to the NICU. By using these diagnoses, and adding parameters resulting from a search of literature, a set of risk parameters can be created. This set will be used to retrospectively analyse the nursing files of infants re-admitted to the NICU within $72 \mathrm{~h}$ after discharge. The exclusion criteria used were re-admittance for phototherapy, inserting an IV or planned surgical or treatment interventions.

Results From July 2009 to July 2013, 44 infants were re-hospitalised at the NICU. Nineteen infants met the inclusion criteria. Of these 19 infants a (graphical) risk profile could be created showing the physiological (in)stability in the three days before discharge.
Conclusions This study shows that infants have different early warning signs of physiological instability. However this instability can be expressed in a risk score, which might predict the chance that the infants are re-admitted to the NICU. The NICU may thus decide to not discharge the infant for a few more days. Further research on the utilisation of the risk score is needed.

\section{PS-263 DETERMINING THE LEVEL OF AWARENESS ON CANCER IN UNIVERSITY STUDENTS}

${ }^{1} \mathrm{M}$ Kurtuncu, ${ }^{2} \mathrm{~L}$ Utas Akahn, ${ }^{2} \mathrm{~S}$ Celik, ${ }^{1} \mathrm{I}$ Alkan. ${ }^{1}$ Pediatric Nursing, Bulent Ecevit University, Zonguldak, Turkey; ${ }^{2}$ Psychiatric Nursing, Bulent Ecevit University, Zonguldak, Turkey

\subsection{6/archdischild-2014-307384.563}

Background and aim The most important reason for the high mortality rates of cancer is the low level of awareness, which leads to a late diagnosis and the treatments starting too late. For this reason, it is important for individuals, especially at younger ages, to gain awareness and integrate taking the necessary precautions into their lifestyle in order to prevent cancer and ensure early diagnosis. This study was performed with the goal of determine the levels of awareness of students studying in two different campuses of a university in the Western Black Sea Region on cancer and its symptoms.

Methods This descriptive/cross sectional study was performed in a university in the Western Black Sea Region between 01. February.2013-01. April.2013. Students studying in the 2012-2013 academic year in a university in the Western Black Sea Region formed the universe of the study. The study was performed in two separate campuses belonging to the university.

Results The average age of the students was $22.01 \pm 3.63$. While female students were the majority in the health sciences campus $(74.8 \%)$, male students were the majority in the central campus (76.5\%). 57.5\% were male. $9.6 \%$ of the students from the health sciences campus and $12.4 \%$ of the students from the central campus thought that cancer was communicable, while most of the students from both campuses knew that smoking caused cancer. It can also be seen that the rate of answering questions regarding cancer correctly was higher among students studying in the health sciences campus.

Conclusion It was determined that students who do not study at the health campus have insufficient information on cancer, cancer symptoms, and the presence of breast cancer in males and approximately half of them regarded obesity as a risk factor for cancer.

\section{PS-264 COMPARISON OF TWO MANUAL VENTILATION DEVICES ON THE PAEDIATRIC INTENSIVE CARE: THE SELF-INFLATING BAG VERSUS THE FLOW-INFLATING BAG}

M de Neef, GJ Muller, AS Kraenzlin, RM Wosten-van Asperen. PICU, Emma Children's Hospital/Academic Medical Center, Amsterdam, Netherlands

\subsection{6/archdischild-2014-307384.564}

Objective Different types of devices for manual (bag) ventilation are available. But which device is the best to use is still a matter of controversy. Most frequently used devices in Dutch PICU's include the Self Inflating Bag (SIB) and the Flow Inflating Bag (FIB). The choice of the system seems to be subjective, and depend mostly on unit policy or personal preferences. Studies 
on manual ventilation in the paediatric population are scarce. We therefore conducted a prospective, observational study to compare both devices with regard to peak pressures generated and consequences on respiratory parameters during manual ventilation of intubated and mechanically ventilated children.

Setting A paediatric intensive care unit of an university hospital. Patients Patients admitted to the PICU; intubated with tube size $3.0,3.5$ or 4 and mechanically ventilated were eligible for the study.

Interventions Manual ventilation was performed with two devices, a Laerdal Silicone paediatric circuit and a Mapleson C cirucuit. After inclusion, a tap was placed at the end of the manual inflation bag tube allowing for continuous pressure measurements during manual ventilation. Subjects were blinded to pressure recording and no feedback was provided. From each measurement a maximum peak pressure, mean peak pressure $(+\mathrm{SD})$, median peak pressure and a frequency was calculated.

Main

Results 412 measurements in 39 patients were performed (205/ 207). Both groups had significant higher peak pressures and median delivered pressure during manual ventilation (for both groups $\mathrm{p}=.000$ ) when compared to ventilator settings.

Discussion This study demonstrates that in order to perform $\mathrm{MH}$ in a safe and effective manner the pressures and volumes generated have to be monitored.

\section{Nutrition, Growth and Morbidity}

\section{PS-265 INSULIN TREATED HYPERGLYCAEMIA, HYPERALIMENTATION AND METABOLIC CHANGES ASSOCIATED WITH GROWTH IN VERY PRETERM INFANTS RECEIVING PARENTERAL NUTRITION}

J Green, L Burgess, C Morgan. Neonatology, Liverpool Women's Hospital, Liverpool, UK

\subsection{6/archdischild-2014-307384.565}

Background Hyperalimentation using a Standardised, Concentrated, Added Macronutrients Parenteral (SCAMP) nutrition regimen improves early head growth, particularly in association with insulin-treated hyperglycaemia (ITH). Early postnatal growth is associated with increased potassium/phosphate supplementation and lower plasma amino acids (AA). We hypothesised that infants randomised to SCAMP and undergoing ITH would demonstrate metabolic changes associated with growth.

Methods SCAMP and control groups were identified from the previously published RCT (ISRCTN 76597892). Infants were substratified into ITH and non-ITH within their original group

\begin{tabular}{lllllll}
\multicolumn{3}{c}{ Abstract PS-265 Table 1} \\
\hline & SCAMP & \multicolumn{5}{c}{ Control } \\
& ITH & Non-ITH & & ITH & Non-ITH & \\
MDI (d $\left.\mathrm{d}^{3}-14\right):$ & $(\mathrm{n}=34)$ & $(\mathrm{n}=34)$ & $\mathrm{p}$ & $(\mathrm{n}=29)$ & $(\mathrm{n}=42)$ & $\mathrm{p}$ \\
Protein $(\mathrm{g} / \mathrm{kg} / \mathrm{d})$ & $3.45(0.37)$ & $3.52(0.27)$ & 0.38 & $2.84(0.20)$ & $2.87(0.24)$ & 0.58 \\
Energy (kcal/kg/d) & $95.8(9.3)$ & $99.2(6.2)$ & 0.13 & $84.9(6.7)$ & $86.4(7.6)$ & 0.36 \\
$\Delta$ OFC at d14 (mm) & $14(6)$ & $11(5)$ & 0.03 & $10(6)$ & $10(6)$ & 0.94 \\
Psupp (infants) & $32(94 \%)$ & $16(47 \%)$ & $<0.01$ & $19(66 \%)$ & $14(33 \%)$ & $<0.01$ \\
Ksupp (infants) & $23(68 \%)$ & $10(29 \%)$ & $<0.01$ & $9(31 \%)$ & $7(17 \%)$ & 0.25 \\
\hline
\end{tabular}

randomisation. Actual mean daily protein/energy intake, weekly growth and secondary metabolic outcome data, including daily electrolyte intake (including supplementary electrolytes), daily plasma electrolyte and weekly AA (profile: 23 individual AA plasma levels) were collected.

Results Table 1 shows mean (sd) daily intake (MDI) protein/ energy intake. The change $(\Delta)$ in OFC data demonstrate improved growth with ITH/SCAMP. The percentage of infants requiring electrolyte supplementation (Ksupp/Psupp) was higher in ITH versus non-ITH infants. 17/23 plasma AA were lower in SCAMP ITH versus non-ITH infants ( $p<0.05$ for cysteine, arginine, glutamine, tryptophan, tyrosine, ornithine, methionine) whereas only 5/23 AA were lower in control ITH versus nonITH (ns).

Conclusions ITH, hyperalimentation and growth is associated with increased potassium/phosphate supplementation and lower plasma AA.

\section{PS-266 HYPERALIMENTATION AND BLOOD GLUCOSE CONTROL IN VERY PRETERM INFANTS: THE RANDOMISED CONTROLLED SCAMP NUTRITION STUDY}

C Morgan, L Burgess, M Grosdenier, P McGowan, MA Turner. Neonatology, Liverpool Women's Hospital, Liverpool, UK

10.1136/archdischild-2014-307384.566

Background The Standardised, Concentrated with Added Macronutrients Parenteral (SCAMP) nutrition regimen is a method of hyperalimentation shown to improve early head growth in very preterm infants. Early hyperalimentation is associated with marked hyperglycaemia. Increasing early protein intake reduces the risk of insulin-treated hyperglycaemia. We hypothesised that increasing carbohydrate and protein intake simultaneously using the SCAMP regimen would not result in more insulin-treated hyperglycaemia.

Methods Control parenteral nutrition (PN) was started within 6 $\mathrm{h}$ birth and infants ( $<1200 \mathrm{~g} ;<29$ weeks) were randomised to start SCAMP or remain on control. Actual nutritional intake, mean blood glucose (MBG) and insulin use data were collected for each day, d1-14.

Results Infants were randomised $\left(\mathrm{d}^{2}-5\right)$ to SCAMP ( $\left.\mathrm{n}=74\right)$ and control $(\mathrm{n}=76)$ groups. In 14-day-survivors (Table 1 ), SCAMP $(n=68)$ achieved higher mean actual protein/carbohydrate intakes than control $(n=72)$ from day $3-4$. This was not associated with a difference in MBG or insulin use.

Conclusion The SCAMP regimen did not result in the major disturbances in blood glucose control previously described with hyperalimentation. We propose this is partly explained by differences in early protein intake.

Abstract PS-266 Table 1 Difference (SCAMP minus control) in mean daily protein/carbohydrate intake, MBG and number of infants requiring insulin

\begin{tabular}{lllllll}
\hline Difference in: & $\mathrm{d} 1-2$ & $\mathrm{~d}^{3}-4$ & $\mathrm{~d} 5-6$ & $\mathrm{~d} 7-8$ & $\mathrm{~d} 9-10$ & $\mathrm{~d} 11-12$ \\
Protein $(\mathrm{g} / \mathrm{kg} / \mathrm{d})$ & 0.05 & $0.21^{* *}$ & $0.78^{* *}$ & $0.81^{* *}$ & $0.62^{* *}$ & $0.58^{* *}$ \\
CHO $(\mathrm{g} / \mathrm{kg} / \mathrm{d})$ & 0.13 & $0.89^{* *}$ & $1.91^{* *}$ & $1.62^{* *}$ & $1.64^{* *}$ & $1.63^{* *}$ \\
Insulin use (infants) & -2 & 1 & -1 & 4 & -2 & 0 \\
MBG (mmol/l) & 0.1 & 0.2 & 0.6 & 0.4 & 0.2 & 0.1 \\
MBG $(95 \% \mathrm{Cl})$ & $(-0.9-1.0)$ & $(-1.0-1.4)$ & $(-0.6-1.8)$ & $(-0.5-1.3)$ & $(-0.8-1.1)$ & $(-0.7-0.9)$ \\
\hline${ }^{*} \mathrm{p}<0.05{ }^{* *} \mathrm{p}<0.01, \mathrm{Cl}$ confidence interval
\end{tabular}

